\title{
Effect of the Electrolyte Surface Tension on the Single Bubble Sono-Luminescence (SBSL)
}

\author{
Seiichiro NAKABAYASHI* and Hideo YANAGITA \\ Department of Chemistry, Faculty of Science, Saitama University (Urawa, Saitama 338-8570, Japan)
}

Received June 15, 1999 ; Accepted August 31, 1999

\begin{abstract}
The laser scattering and the light emission from a single stable sonoluminescing bubble were observed in the pure water and $\mathrm{NaCl}$ aqueous solution. The emission intensity was enhanced in $1 \mathrm{M}\left(\mathrm{M}=\mathrm{mol} \mathrm{dm}^{-3}\right) \mathrm{NaCl}$ electrolyte under higher acoustic pressures but the time course of the scattering intensity was not affected. These findings were discussed in terms of the effect of the surface energy at the interface of the bubble.
\end{abstract}

Key Words : Surface Tension, SBSL

\section{Introduction}

A liquid interface is the one of the most attractive topic in the modern aspects of electrochemistry. ${ }^{1)}$ The structure and the reactivity of the electrolyte surface in contact with an air or a mutually immiscible liquid has been studied extensively by using a non-linear laser spectroscopy coupled with electrochemical techniques. ${ }^{2)}$ These experiments have led to a better understanding of the stationary characteristics of the interface. However, at the interface deforming its shape with a high velocity, there still remains a lot of unexplained phenomena. Among them, the most exotic one is the light emitting bubble while it undergoes highly non-linear radial pulsation. Ultrasonic standing wave in pure water induces a light emission from a microscopic single bubble. This is so-called single bubble sono-luminescence (SBSL), which was discovered by Gaitan in 1990..-5) The exciting features of this phenomenon are; (1) the light emission is bunched into a short pulse whose duration is within a few pico-second. ${ }^{9,10)}$ (2) the emission spectrum is broad and does not have any specific line structure. The spectrum can be fitted to the black body emission and the estimated temperature at the emission center is over $30000 \mathrm{~K}^{6-8)}$

Probably, by historical reason, this exciting phenomenon has been studied in the hydrodynamic and/ or ultrasonic communities. ${ }^{11)}$ However, SBSL must be much attractive from the chemical and also the electrochemical point of view; for example, the bubble is the very small chemical reactor whose temperature is over $30000 \mathrm{~K}$. The surface of the bubble; the interface between the liquid and the gas phases, changes its shape with an extremely high speed. In this article, we have forced our attention into the time dependent behavior of the interface when the bubble collapses. The addition of a salt in the aqueous solution enhances the stability of the bubble when it collapses under the higher acoustic pressure and increases the intensity of the emission.

\section{Experimental}

The experimental setup is schematically shown in Fig. 1. The $100 \mathrm{ml}$ round bottom flask contained degassed water. The water was degassed perfectly by continuous boiling for at least 1.5 hours. After that, the temperature was cooled down to the room temperature. This degassed process was critical in order to obtain the stable SBSL. A couple of PZT (lead zirconate titanate) oscillators were attached symmetrically on the both sides of the flask. The oscillators were driven at $c a .46 \mathrm{kHz}$, which was decided from the sound velocity of $1471 \mathrm{~m} / \mathrm{s}$ in the water at $290 \mathrm{~K}$ to produce the standing wave with a couple of nodes in the flask. In order to seed a nuclei of the bubble, the air was introduced from a pipette at the initial stage. At the bottom of the flask, a small PZT was attached in order to pick up the acoustic standing wave in the flask.

The emission was recorded by an image intensified CCD camera(PentaMAX EEV, 512x1024 FT) with COCINA-TV LENS $(f=25 \mathrm{~mm}, \mathrm{~F}=1.4)$. Thus, the $2 \mathrm{D}$ image of SBSL was obtained in a computer.

The time course of the bubble radius was measured by a laser light scattering intensity using a photomultiplier tube coupled with a wide band oscil-

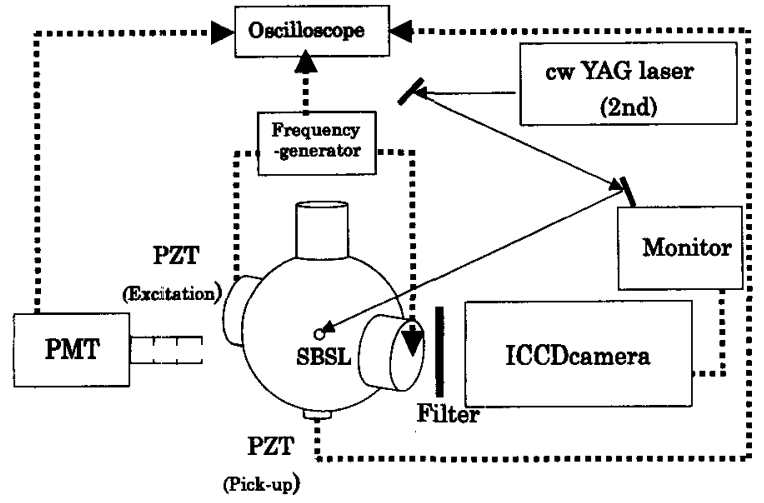

Fig. 1 Experimental setup for measuring the emission intensity from SBSL and the time course of the bubble radius. 
loscope. The bubble was irradiated by a frequency doubled CW YAG laser (Coherent DPSS532), and the wavelength of the emission was $532 \mathrm{~nm}$.

\section{Results and Discussion}

The SBSL in the flask was demonstrated in Fig. 2. The white small dot at the center of the flask was the SBSL. The color of the emission was blue-white. In order to show the shape of the flask, the trace of the room light was introduced into the light shielding box. The white area at the right side of the flask was the reflection of this room light. The spatial distribution of the emission intensity was shown by the lines located at the left and the bottom of the picture. The peaks at $250 \mathrm{ch}$ on the horizontal axis (bottom in the figure) and at $290 \mathrm{ch}$ on vertical axis (left) are form the SBSL. The others such as at $300-400 \mathrm{chs}$ on the horizontal axis are from the room light. When the emission intensity from SBSL was measured, the system was perfectly shielded from the room light. The emission of SBSL was stable for at least 60 minuets spatially and also temporally. The emission intensity was obtained by averaging the SBSL image for $0.5 \mathrm{~s}$.

The time courses of the laser scattering intensity and the acoustic pressure were shown in Fig. 3 (a) and (b), respectively. The scattering intensity is proportional to the radius of the bubble. ${ }^{12)}$ The bubble grew gradually under the negative acoustic pressure, and it shrank quickly when the pressure turned into positive. The light pulse was obtained when the bubble was collapsed and after this collapsing, the dumping oscillation of the bubble radius was observed. The collapsing of the bubble occurred much quicker than the bubble growth. The actual size of the bubble can be estimated by the shadow-graph method. ${ }^{12)}$ The maximum radius of the bubble was roughly estimated to be ca. $70 \mu \mathrm{m}$ in this experimental conditions. ${ }^{12)}$ The time profile of the laser scattering intensity was not modified by the addition of $\mathrm{NaCl}$ upto the concentration of $1 \mathrm{M}$.

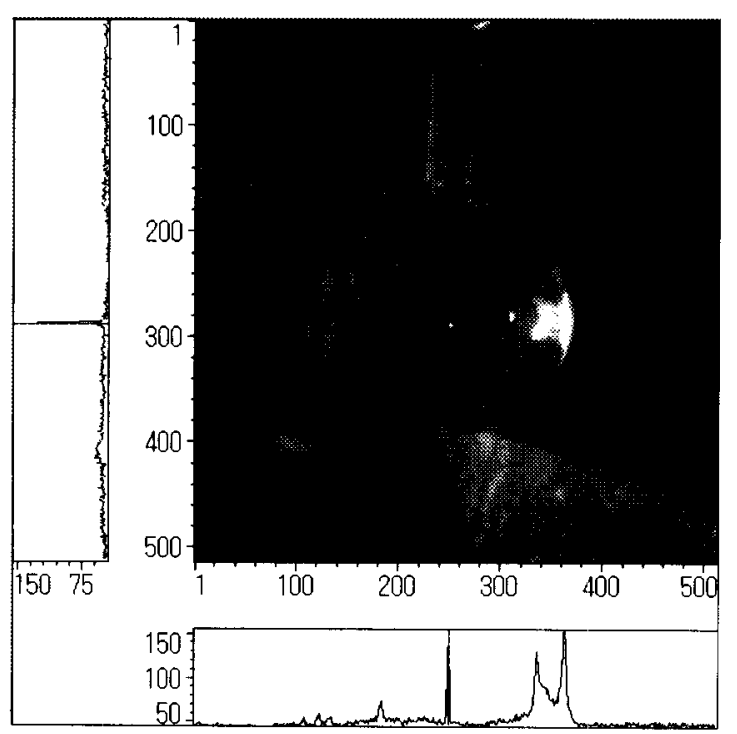

Fig. 2 Typical picture of the SBSL in a round-bottle flask.

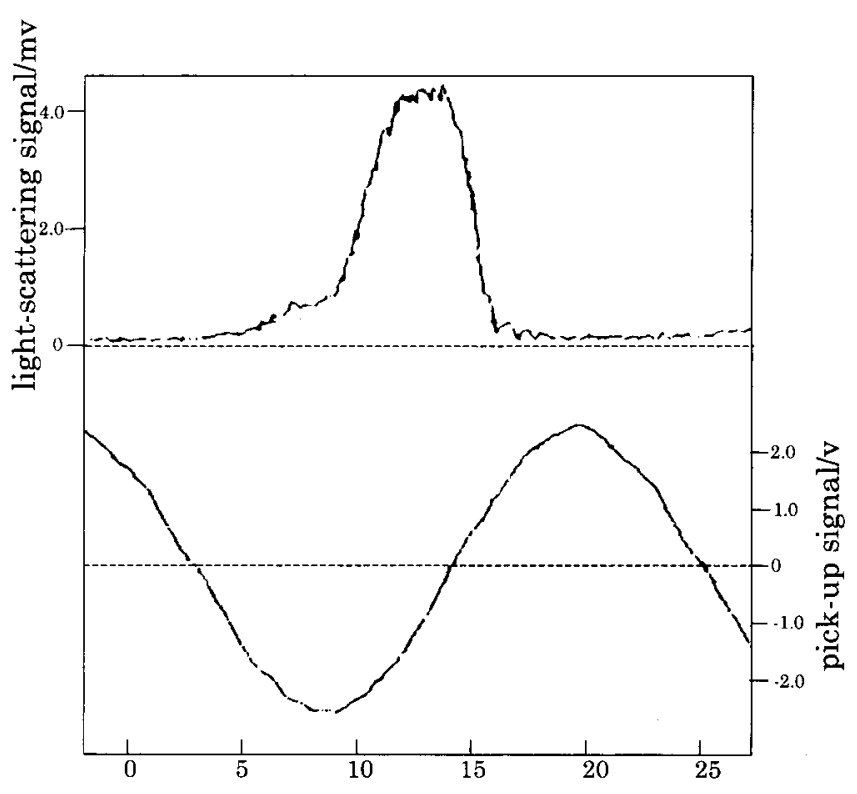

Time $/ \mu$ sec

Fig. 3 Time course of the scattering intensity which is proportional to the bubble radius (a), and the acoustic pressure obtained by a piezoelectric disk at the bottom of the flask (b).

In order to estimate the energy accumulation in the bubble when it collapses, the contribution from the surface energy is not negligibly small when the particle size is less than $10 \mu \mathrm{m}{ }^{13,14)}$ The inner pressure of the bubble, $P_{\text {in }}$ is known to be larger than the outer pressure of the bubble, $P_{\text {out }}$ as

$$
P_{\text {in }}=P_{\text {out }}+2 \sigma / r
$$

where $r$ is a radius of the bubble and $\sigma$ is a surface tension of the interface. ${ }^{13,14)}$ When the area of the interface changes, the heat formation accompanies as

$$
Q=-T(\mathrm{~d} \sigma / \mathrm{d} T) \mathrm{d} A
$$

where $Q, T$ and $A$ are the heat, the absolute temperature and the surface area, respectively..$^{13,14)}$

While the water temperature was kept constant to be $20{ }^{\circ} \mathrm{C}$, the emission intensities were compared among in the pure water and in the electrolyte containing $0.01-1 \mathrm{M} \mathrm{NaCl}$. In the solution of $0.01,0.05$ and $0.1 \mathrm{M}$, the emission intensity was not different from the one obtained in the pure water. However, in the $1 \mathrm{M}$ solution, the intensity increased under higher acoustic pressure. The intensities were plotted as a function of the driving voltage of the PZT as shown in Fig. 4. At this stage, although the accurate value of the acoustic pressure was not known, the pressure was estimated $1.3-1.5 \mathrm{~atm}$ by the height of the signal from the pick-up PZT when the electric input was 16 $\mathrm{V}$. The acoustic pressure is known to be proportional to the driving voltage of the PZT. ${ }^{12)}$ The emission intensities increased as a function of the acoustic pressure. In the higher pressure region, the one in $1 \mathrm{M}$ electrolyte was systematically enhanced than the one in the pure water. The resonant frequency of the acoustic excitation, which was dependent on the velocity of the sound in the solution was varied from 


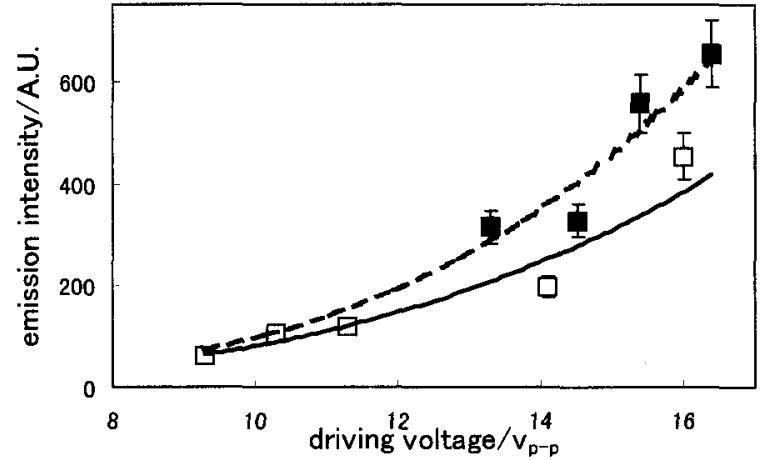

Fig. 4 The relationship between the emission intensities and the driving voltage of the PZT oscillators in the pure water ( $\square$ ) and in $1 \mathrm{M} \mathrm{NaCl}$ aqueous solution

$46.2 \mathrm{kHz}$ to $47.8 \mathrm{kHz}$ by adding $1 \mathrm{M} \mathrm{NaCl}$ in the solution. However, the observed enhancement in the emission was larger than the increase of the flashing number ca, $3 \%$. Then, we can conclude that the emission intensity was enhanced in $1 \mathrm{M} \mathrm{NaCl}$ solution. Although the spectrum of the emission was not measured yet, to the dark-adapted human eyes, the color of the emission was not changed by the addition of $\mathrm{NaCl}$.

The surface tension of an aqueous solution is known to change as the concentration of a salt dissolved in it. When 1-1 electrolyte such as $\mathrm{NaCl}$ is added into the pure water, the surface tension changes as

$$
\sigma=\sigma_{0}+(79.517 c / \varepsilon) \log _{10}\left\{1.143 \times 10^{-13}(\varepsilon \mathrm{T})^{3} / c\right\}
$$

where $\sigma_{0}, \varepsilon$ and $c$ are the surface tension of the pure water, the dielectric constant of the water and the concentration of a solute in mols per liter. ${ }^{15)} \mathrm{T}$ is the absolute temperature. The surface tension of the pure water at $20{ }^{\circ} \mathrm{C}$ is $72.75 \mathrm{erg} / \mathrm{cm}^{2}$ and it increases about $3 \%$ by adding $1 \mathrm{M} \mathrm{NaCl}$. The ratio $\left(\sigma / \sigma_{0}\right)$ is apparently small, 1.03 . However, because the inner pressure $P_{\text {in }}$ increases as a function of $(\sigma / r)$ as shown in eq.(1), the difference in the accumulation energy in the bubble must be enhanced by the P.V term when the bubble radius becomes small. This is consistent with the observation that the laser scattering was almost constant by adding $\mathrm{NaCl}$. These results suggest that the energy charge into the bubble from the surface is affected by the P-V work term estimated by eq. (1) rather than by the $\sigma$-A work term by eq.(2).

\section{Acknowledgment}

This work has been partly supported by Grant-in Aid for Scientific Research No.90554042 and on Priority Area of Electrochemistry of Ordered Interfaces from Ministry of Education, Science, Sports and Culture, Japan.

\section{References}

1) A. G. Volkov and D. W. Deamer, Eds, Liquid-Liquid Interfaces. Theory and Methods, CRC Press, New York, N. Y. (1996).

2) K. B. Eisenthal, Acc. Chem. Res., 26, 636 (1993).

3) D. F. Gaitan, L. A. Crum, C. C. Church, and R. A. Roy, J. Acoust. Soc. Am., 91, 3166 (1992).

4) D. F. Gaitan, A. A. Atchley, S. D. Lewis, J. T. Carlson, X. K. Maruyama, M. Moran, and D. Schweider, Phys. Rev. E, 54, 525 (1996).

5) B. P. Baber and S. J. Putterman, Nature, 352, 318 (1991).

6) B. P. Baber, R. A. Hiller, R. Lofstedt, S. T. Putterman, and K. R. Weninger, Phys. Report, 281, 65 (1997).

7) K. Yasui, Phys. Rev. E, 58, 471 (1998).

8) T. Kokubun, S. Hayashi, Q. Han, and T. Kamakura, J. J. Appl. Phys., 37, 5835 (1998).

9) R. Pecha, B. Gompf, G. nick, Z. Q. Wang, and W. Eisenmenger, Phys. Rev. Lett., 81, 717 (1998).

10) R. A. Hiller, S. T. Putterman, and K. R. Weninger, Phys. Rev. Lett., 80, 1090 (1998).

11) L. A. Crum, T. J. Mason, J. L. Reisse, and K. S. Suslick, Eds, Sonochemistry and Sonoluminescence, Kluwer, London (1999).

12) T. R. Stottlemyer and R. E. Apfel, J. Acoust. Soc. Am., 102, 1418 (1997).

13) D. H. Everett, Basic Principles of Colloid Science, Royal Society of Chemistry, Piccadilly, London (1988).

14) A. W. Adamson, Physical Chemistry of Surfaces, 2nd edn, Interscience Pub., N.Y. (1967).

15) L. Onsager and N. N. T. Samaras, J. Chem. Phys., 2, 528 (1934). 